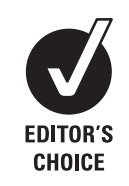

See Editorial, p 1233

${ }^{1}$ Department of Pathology, University Medical Centre Utrecht, The Netherlands; ${ }^{2}$ Department of Surgery, Onze Lieve Vrouwe Gasthuis, Amsterdam, The Netherlands

Correspondence to:

Ms C H M van Deurzen,

Department of Pathology, University Medical Centre

Utrecht, P0 Box 85500, 3508 GA Utrecht, The Netherlands; c.vandeurzen@umcutrecht.nl

Accepted 12 August 2008 Published Online First 15 September 2008

\title{
In-transit lymph node metastases in breast cancer: a possible source of local recurrence after Sentinel Node procedure
}

\author{
C H M van Deurzen, ${ }^{1}$ P J Borgstein, ${ }^{2}$ P J van Diest ${ }^{1}$
}

\begin{abstract}
Aims: In-transit lymph node metastases are a common phenomenon in melanoma patients and have been increasingly recognised since the introduction of the Sentinel Node (SN) procedure. To which extent this also occurs in patients with breast cancer has not been studied yet. The aim of this study was therefore to explore the occurrence of in-transit lymph node metastases in patients with breast cancer.
\end{abstract}

Methods: Afferent lymph vessels to the SN identified by blue dye were removed from 17 patients with breast cancer during a regular SN procedure.

Results: Three out of 17 patients showed a lymph node associated with the afferent lymph vessel. One of these lymph nodes showed a breast cancer macrometastasis, to be regarded as an in-transit metastasis. This metastasis would normally have been left in situ. Conclusions: In-transit lymph nodes associated with the afferent SN lymph vessels seem to occur in a significant proportion of patients with breast cancer. These lymph nodes may contain metastases, which are a potential source of local recurrence when left in situ. This finding generates the hypothesis that there may be an indication to remove these lymph vessels during the SN procedure.

The sentinel node (SN) is the first lymph node to receive lymphatic flow from the primary tumour and is therefore the first to contain metastatic tumour cells. ${ }^{2}$ The combination of preoperative lymphoscintigraphy and an intraoperative handheld detecting probe allows detection of the SN in nearly all patients with breast cancer. ${ }^{34}$ Lymph nodes that lie in close contact with a lymphatic channel between the primary tumour and a regional lymph node basin are called in-transit, interval or intercalated lymph nodes. There can be a single or several in-transit nodes along the course of a lymphatic vessel. As these might be small, they may not be recognised as SNs, although they may receive direct lymphatic drainage from the primary tumour and may thus in fact be the first lymph node to be colonised with tumour cells. When these metastatic cells grow out, such a lymph node metastasis may become clinically manifest.

The concept of in-transit metastases is especially known from melanoma studies that have reported an incidence of in-transit nodes between 3 and 10 $\% .^{5-8}$ Metastatic deposits were identified in $14-20 \%$ of these cases, and in some cases the in-transit node was the only site of metastases. These nodes are a recognised site of metastatic recurrence after an SN procedure in melanoma patients. ${ }^{9}$ In-transit nodes and their potential to harbour metastases have to our knowledge not been studied in breast cancer, which was the aim of the present study.

\section{MATERIALS AND METHODS \\ Patients}

A group of 17 clinically node-negative female patients with breast cancer underwent a lumpectomy or a mastectomy and an usual SN procedure applying dynamic lymphoscintigraphy (with radiolabelled colloid) and intradermal Patent blue dye (Guerbet, Aulnay-sous-Bois, France). During surgery, the afferent, blue-stained axillary lymph vessels were identified and excised with some surrounding fatty tissue by an experienced breast cancer surgeon (PB). An axillary lymph node dissection (ALND) was performed at a later date when metastatic disease was found in the SN. Table 1 shows descriptive characteristics of the study population.

\section{Histopathology}

The excised afferent lymph vessels were neutral buffered formaldehyde fixed and completely embedded in paraffin. Five micrometre thick stepsections were cut parallel to their longitudinal axis at $200 \mu \mathrm{m}$ intervals through the complete block for an initial staining with haematoxylin and eosin $(\mathrm{H} \& \mathrm{E})$, keeping a blank ribbon at each level for immunohistochemistry (IHC) with CAM 5.2 (dilution 1:80; Beckton Dickinson Biosciences, San Jose, CA) in case in-transit nodes were identified. In transit nodes were initially screened by $\mathrm{H} \& \mathrm{E}$ staining, and one level of IHC was conducted in case a metastasis was detected in the H\&E staining. In nodes where no tumour was seen in the H\&E staining, IHC was performed on all available levels (up to 7). The SNs removed in the same session were processed according to the protocols described previously. ${ }^{10} 11$

\section{RESULTS}

Histopathological analysis of the excised afferent lymph vessels (median length $2.5 \mathrm{~cm}$; range 2.0 $3.0 \mathrm{~cm}$ ) showed well-developed lymph vessels surrounded by mature adipose tissue. Three patients showed one small perilymphatic lymph node each (diameters 3.3, 4.5 and $9.0 \mathrm{~mm}$, respectively, to a total of $3 / 17(18 \%))$. One of these lymph nodes showed a breast adenocarcinoma macrometastasis (size $3.4 \mathrm{~mm}$ ) without extranodal extension, confirmed by immunohistochemistry (fig 1). This patient also had a tumour-positive axillary $\mathrm{SN}$ macrometastasis (diameter metastasis: $2.4 \mathrm{~mm}$, total lymph node diameter: $14 \mathrm{~mm}$ ) 

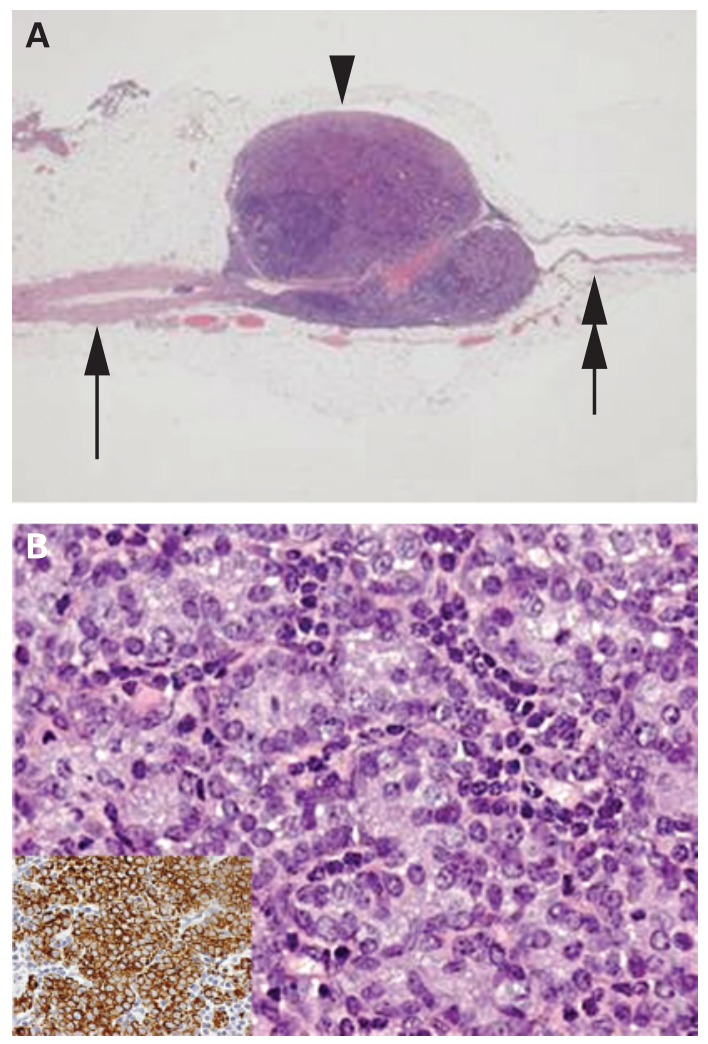

Figure 1 (A) In-transit lymph node surrounding the afferent lymph vessel $(\mathrm{H \& E}$, original magnification $\times 1)$. Arrowhead: metastatic tumour deposit. Single arrow: afferent lymph vessel. Double arrow: efferent lymph vessel. (B) Metastatic tumour deposit (H\&E, original magnification $\times 20$ ) with immunohistochemical confirmation (CAM 5.2; inset).

which was followed by an ALND without further metastases. A re-excision specimen 2 years after breast-conserving therapy because of a mammographically suspect lesion showed scarring and calcification, but no local recurrence in this patient. The other two in-transit lymph nodes showed fatty degeneration, but no tumour cells (confirmed by CAM 5.2). One of these patients had a tumour-positive axillary SN (diameter metastasis: $4.9 \mathrm{~mm}$, total lymph-node diameter: $15 \mathrm{~mm}$ ), which was followed by an ALND without any additional lymph nodes involved.

\section{DISCUSSION}

In-transit recurrence is an important morbidity factor in melanoma patients. This phenomenon has not yet been reported in patients with breast cancer. The probability of developing in-transit metastases in patients with breast cancer is important, since it could occasionally explain a local recurrence.

In our study, three patients with breast cancer showed a lymph node in close relationship with the SN afferent lymph vessel. Therefore, these lymph nodes may be the first lymph nodes draining the tumour area and so could theoretically be called the "true" SNs. However, none of these in-transit nodes were detected using lymphoscintigraphy or by the handheld gamma probe, even after excision of the lymphatic duct tissue samples. There may be several explanations for this. First, replacement of the true SN by a large metastatic deposit could cause a mechanical obstruction, which deviates the lymphatic flow including radioactive tracer and blue dye towards a false
Table 1 Clinicopathological characteristics of 17 patients with breast cancer undergoing a Sentinel Node biopsy and resection of the afferent lymph vessels

\begin{tabular}{ll}
\hline Feature & No \\
\hline Tumour size (median) & $1.5 \mathrm{~cm}$ \\
Tumour type & 13 \\
$\quad$ Ductal & 2 \\
Lobular & 1 \\
$\quad$ Mixed ductal and lobular & 1 \\
$\quad$ Encysted papillary carcinoma* & \\
Histological grade & 3 \\
1 & 13 \\
2 & 0 \\
3 & \\
Steroid receptor status & 15 \\
Estrogen receptor positive & 13 \\
Progesteron receptor positive $\dagger$ & \\
No of positive SNs & 10 \\
0 & 7 \\
1 &
\end{tabular}

*Encysted papillary carcinoma, no infiltrative growth pattern. †PR status was unknown in one patient.

"SN." This may have occurred in one of our three in-transit nodes. Second, tracer uptake could also be low in case of strong fatty degeneration of these lymph nodes, as we observed in two of our three in-transit nodes. Third, these in-transit nodes are small and located near the breast, so they may easily escape lymphoscintigraphy, palpation and gamma probe detection, since the in-transit signal may, due to proximity, not be distinguished from the primary injection signal.

In our series, one patient had an in-transit lymph node metastasis of $3.4 \mathrm{~mm}$ in size, which is therefore a macrometastasis. Since this patient also had a metastasis in the SN, this was of no further therapeutic significance for this patient, as she underwent an ALND and received adjuvant therapy. However, if left in situ, this in-transit node metastasis could well have been a source of local recurrence, although locoregional radiotherapy as a standard part of breast-conserving therapy may help to prevent outgrowth of such in-transit node metastases. Nevertheless, one can imagine that in some patients, the in-transit node may be the only lymph node harbouring metastases, and as these may be macrometastases, as in the present study, this may be of clinical significance.

In conclusion, in-transit lymph nodes associated with the afferent SN lymph vessels seem to occur in a significant proportion of patients with breast cancer, and may contain (macro) metastases. These metastases are a potential source of local recurrence when left in situ. This finding generates the hypothesis that in-transit lymph nodes (and metastases) may have major clinical impact, and there may be an indication to remove the tumour draining lymph vessels during the $\mathrm{SN}$ procedure. However, this hypothesis needs to be substantiated by larger series.

Competing interests: None.

\section{REFERENCES}

1. Giuliano $\mathbf{A E}$, Kirgan DM, Guenther JM, et al. Lymphatic mapping and sentinel lymphadenectomy for breast cancer. Ann Surg 1994;220:391-98; discussion 398401.

2. Krag D, Weaver D, Ashikaga T, et al. The sentinel node in breast cancer-a multicenter validation study. N Engl J Med 1998;339:941-6.

3. Borgstein PJ, Pijpers R, Comans EF, et al. Sentinel lymph node biopsy in breast cancer: guidelines and pitfalls of lymphoscintigraphy and gamma probe detection. J Am Coll Surg 1998;186:275-83. 
4. Pijpers R, Meijer S, Hoekstra OS, et al. Impact of lymphoscintigraphy on sentinel node identification with technetium-99m-colloidal albumin in breast cancer. J Nucl Med 1997;38:366-8.

5. Uren RF, Howman-Giles R, Thompson JF, et al. Interval nodes: the forgotten sentinel nodes in patients with melanoma. Arch Surg 2000;135:1168-72.

6. McMasters KM, Chao C, Wong SL, et al. Interval sentinel lymph nodes in melanoma. Arch Surg 2002;137:543-47; discussion 547-9.

7. Thelmo MC, Morita ET, Treseler PA, et al. Micrometastasis to in-transit lymph nodes from extremity and truncal malignant melanoma. Ann Surg Oncol 2001;8:444-8.
8. Vidal-Sicart S, Pons F, Fuertes S, et al. Is the identification of in-transit sentinel lymph nodes in malignant melanoma patients really necessary? Eur J Nucl Med Mol Imaging 2004;31:945-9.

9. Statius Muller MG, van Leeuwen PA, van Diest PJ, et al. Pattern and incidence of first site recurrences following sentinel node procedure in melanoma patients. World J Surg 2002;26:1405-11.

10. van Diest PJ. Histopathological workup of sentinel lymph nodes: how much is enough? J Clin Pathol 1999;52:871-3.

11. van Diest $\mathbf{P J}$, Peterse HL, Borgstein PJ, et al. Pathological investigation of sentinel lymph nodes. Eur J Nucl Med 1999;26(4 Suppl):43-9S.

\section{Let us assist you in teaching the next generation}

Figures from all articles on our website can be downloaded as a PowerPoint slide. This feature is ideal for teaching and saves you valuable time. Just click on the image you need and choose the "PowerPoint Slide for Teaching" option. Save the slide to your hard drive and it is ready to go. This innovative function is an important aid to any clinician, and is completely free to subscribers. (Usual copyright conditions apply.) 